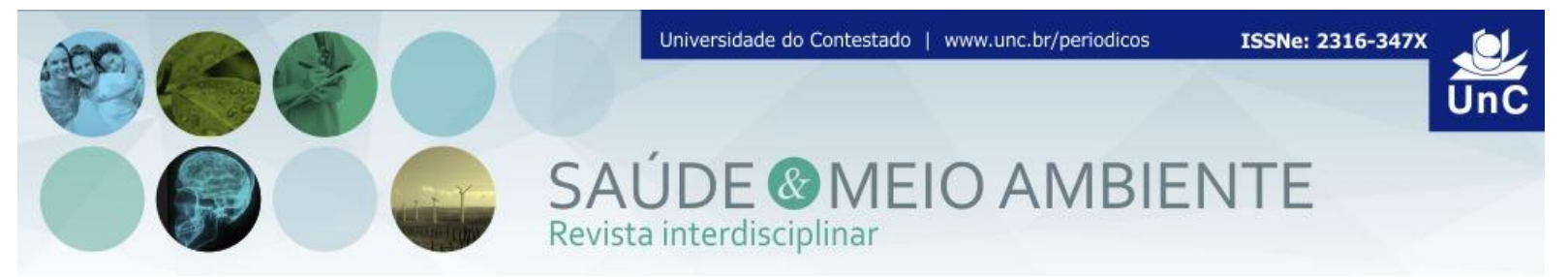

\title{
GERENCIAMENTO DE RESÍDUOS EM SERVIÇOS DE SAÚDE DO SERTÃO NORDESTINO
}

\author{
Ana Maria Machado Borges ${ }^{1}$ \\ José Erickson do Nascimento Timóteo ${ }^{2}$ \\ Milenna Alencar Brasil ${ }^{3}$ \\ Alessandra Bezerra de Brito ${ }^{4}$ \\ Antônio Germane Alves Pinto ${ }^{5}$ \\ Fernando Luiz Affonso Fonseca ${ }^{6}$
}

\begin{abstract}
RESUMO
O descarte inadequado dos resíduos de serviços de saúde pode afetar o ambiente pelo potencial de contaminação desse material. A pesquisa teve a seguinte pergunta de partida: como é realizado o processo de segregação e acondicionamento de Resíduos de Serviços de Saúde (RSS) no Município de Cedro, Pernambuco, nordeste do Brasil? O estudo teve como objetivo identificar os procedimentos adotados pelos profissionais no processo de segregação e acondicionamento de Resíduos de Serviços de Saúde. Pesquisa qualitativa, realizada nos serviços de saúde de Cedro, Pernambuco. Aplicou-se questionário à equipe de enfermagem. As narrativas foram analisadas pela análise de conteúdo. Evidencia-se que os procedimentos adotados pelos profissionais são feitos de maneira inadequada. Os relatos mostram que há mistura de resíduos comuns e infectados. A organização dos recipientes para descarte é improvisada e fora dos padrões técnicos. Conclui-se que há necessidade de capacitação para qualificar os processos de gerenciamento dos resíduos.
\end{abstract}

Palavras-chave: Resíduos de Serviços de Saúde. Gerenciamento de Resíduos. Equipe de Enfermagem. Atenção Primária à Saúde. Promoção da Saúde.

\footnotetext{
${ }^{1}$ Mestranda em Ciências da Saúde. Especialista em Administração Hospitalar e Sistemas de Saúde. Especialista em Docência do Ensino Superior. Faculdade Leão Sampaio. Faculdade de Medicina do ABC. Hemocentro Regional do Crato. Ceará. Brasil. E-mail: anaborges@leaosampaio.edu.br

${ }^{2}$ Enfermeiro, graduado pela Faculdade Leão Sampaio, Juazeiro do Norte, Ceará, Brasil. E-mail: ericksontimoteo@hotmail.com

${ }^{3}$ Mestre em Ensino na Saúde pela Universidade Estadual do Ceará. Professora do curso de enfermagem da Faculdade Leão Sampaio, Juazeiro do Norte, Ceará, Brasil. E-mail: milena@leaosampaio.edu.br

${ }^{4}$ Especialista em Docência do Ensino Superior pela Faculdade Leão Sampaio. Professora do curso de enfermagem da Faculdade Leão Sampaio, Juazeiro do Norte, Ceará, Brasil. E-mail: alessandrabrito@leaosampaio.edu.br

${ }^{5}$ Doutor em Saúde Coletiva pela Universidade Estadual do Ceará. Professor do curso de enfermagem da Universidade Regional do Cariri, Crato, Ceará, Brasil. E-mail: germanepinto@hotmail.com

${ }^{6}$ Doutor em Medicina (Hematologia) pela Faculdade de Medicina da Universidade de São Paulo. Orientador Permanente do Programa de Mestrado e Doutorado da Faculdade de Medicina do ABC. São Paulo. Brasil. E-mail: profferfonseca@gmail.com
} 


\title{
WASTE MANAGEMENT OF HEALTH SERVICES IN THE NORTHEASERN HINTERLAND
}

\begin{abstract}
Inadequate management of medical waste can affect a large number of people. The waste has the potential to contaminate people, animals and the environment. The research had the following start question: as it is performed the process of segregation and packaging of health care waste in the city of Cedro, Pernambuco, northeast of Brazil? The study aimed to identify the procedures adopted by professionals in the segregation process and packaging of medical waste. Qualitative research conducted in Cedro health services, Pernambuco. Applied questionnaire to the nursing staff, analyzing the data through content analysis. The procedures adopted by professionals are not made properly. The reports show that there are mixture of common and infected waste. There is no standardization of containers for disposal and these often are improvised. There is a need to coach the professionals on the theme.
\end{abstract}

Keywords: Medical Waste. Waste Management. Nursing. Team. Primary Health Care. Health Promotion.

\section{INTRODUÇÃO}

O volume e potencial para toxicidade dos resíduos produzidos pela sociedade moderna têm gerado impacto ambiental negativo. São considerados resíduos sólidos aqueles decorrentes de atividades agrícolas, domésticas, comerciais, industriais, de varrição e hospitalares de consistência semissólida e sólida (BRASIL, 2010).

Resíduos de Serviços de Saúde (RSS) são definidos como aqueles resultantes de atividades realizadas por unidades prestadoras de assistência à saúde como Unidades Básicas de Saúde (UBS), hospitais, clínicas médicas, clínicas odontológicas e laboratórios, assistindo os usuários em suas necessidades médicas, odontológicas, farmacêuticas e laboratoriais, bem como instituições de Ensino e Pesquisa relacionadas à saúde médica e veterinária, que necessitem de processos diferenciados em seu gerenciamento (SALES et al., 2009; PEREIRA et al., 2013).

Embora constitua uma mínima parcela de todo o resíduo produzido em uma comunidade, os RSS oferecem riscos emergentes à segurança de trabalhadores responsáveis por seu manuseio, à qualidade do meio ambiente e à saúde da população em geral, por meio de seus constituintes tóxicos e de difícil degradação (BASU; DAS; PAL, 2012).

São atribuídos aos geradores dos RSS a sua correta manipulação, compreendendo as etapas de coleta, transporte, armazenamento, tratamento e destinação final de seus resíduos. Essa conduta é um meio eficaz para minimizar o 
risco de acidentes, exposição a doenças infectocontagiosas, danos ao meio ambiente, dentre outros (RAMOS et al., 2011).

Para que haja o manejo adequado dos resíduos, a legislação obriga a formulação, por parte dos serviços de saúde, do Plano de Gerenciamento Resíduos de Serviços de Saúde (PGRSS) (BRASIL, 2004). Entende-se por PGRSS um documento que integra o processo de licenciamento ambiental, apontando e detalhando as atividades inerentes aos procedimentos relacionados ao gerenciamento dos RSS nos estabelecimentos de saúde. O planejamento e implementação deste, fica a cargo do responsável legal pelo estabelecimento e sua elaboração deve estar embasada em diretrizes normativas, legais e científicas (GONÇALVES et al., 2011).

O plano tem o propósito de minimização da geração de resíduos e ainda proporcionar aqueles já produzidos um destino seguro, com eficiência e adoção de medidas de segurança e saúde para trabalhadores. Desta forma, o mesmo busca através destas medidas, proteger direta e indiretamente os envolvidos com 0 manuseio, preservar a saúde pública, o meio ambiente e os recursos materiais. Para que de fato as ações sejam efetivadas, devem-se considerar os riscos e características, abrangendo os aspectos pertinentes à geração, segregação, acondicionamento, coleta, armazenamento, transporte, tratamento e disposição final. A elaboração do PGRSS é um processo minucioso que requer responsabilidade, compromisso e dedicação conjunta, de todos os envolvidos nas etapas que vão desde a análise situacional com a identificação do problema até sua implantação no estabelecimento gerador de resíduos (BRASIL, 2004).

É de imensa valia que os profissionais integrantes dos serviços de saúde se empenhem ao máximo na elaboração e execução do plano. As etapas de manejo dos resíduos devem ser executadas da forma como estão estabelecidas no plano. Faz-se necessário compreender a gestão de RSS e agir de maneira resolutiva, embasados na redução, reutilização e a reciclagem, os quais constituem os princípios básicos de um bom gerenciamento.

O gerenciamento dos resíduos no Brasil é precário, devido a fatores como o descaso com estes por parte dos estabelecimentos de saúde, o descarte inadequado, a falta de Equipamentos de Proteção Individual (EPI), bem como o desconhecimento dos riscos pertinentes à exposição destes, por parte de muitos profissionais. O problema se acentua quando se observa que a população também não descarta adequadamente os resíduos domésticos (Marchi et al., 2013). Assim, os resíduos são destinados a ambientes a céu aberto, aterros não controlados, rios e demais lugares onde os meios legais de vigilância não são adotados, elevando seu impacto nocivo (PEREIRA et al., 2013; NASCIMENTO et al., 2009).

Pesquisas recentes realizadas sobre o conhecimento dos profissionais acerca do manejo de resíduos apontam que estes conhecem os procedimentos que devem ser realizados nas etapas gerenciais dos RSS, em especial na segregação e acondicionamento (DOI; MOURA, 2011; SANTOS; SOUZA, 2012). No entanto, pesquisas realizadas sobre os procedimentos adotados pelos profissionais no momento da segregação e acondicionamento revelam que estes não são feitos de 
forma adequada, de acordo com o que preconiza a legislação (ALENCAR et al., 2014; ALVES et al., 2012). A infraestrutura dos serviços de saúde mantém insuficiente ou inexistente a separação dos resíduos de saúde nas unidades (SCHOLZ; NGOLI; FLESSA, 2015).

Ao se realizar a segregação adequada dos resíduos os profissionais estão cumprindo com a legislação e, principalmente, estão adotando medidas em uma fase do gerenciamento de resíduos que é crucial para que todas as outras etapas sejam feitas de maneira adequada, que é a separação dos resíduos na fonte aos quais são gerados e o seu correto acondicionamento.

As unidades básicas de saúde em municípios de pequeno porte, principalmente, a Estratégia de Saúde da Família, realizam uma variedade de atendimentos, que vão desde os procedimentos assistenciais até as ações educativas visando à promoção da saúde e qualidade de vida (BAPTISTINI; FIGUEIREDO, 2014). As atividades assistenciais geram resíduos nessas unidades que necessitam do correto manejo da mesma forma que os resíduos hospitalares. Assim, buscou-se resposta à seguinte indagação: como é realizado o processo de segregação e acondicionamento de Resíduos de Serviços de Saúde (RSS) no Município de Cedro, Pernambuco, nordeste brasileiro?

Para responder a esse questionamento, a pesquisa foi delineada para atingir o seguinte objetivo: identificar os procedimentos adotados pelos profissionais no processo de segregação e acondicionamento de Resíduos de Serviços de Saúde.

\section{METODOLOGIA}

Tratou-se de uma pesquisa descritiva, com abordagem qualitativa.

A pesquisa ocorreu no município de Cedro, Pernambuco, localizado a $569 \mathrm{Km}$ da capital Recife. Possui uma área de $171.640 \mathrm{Km}^{2}$ e uma população estimada em 2013 de aproximadamente 11. 323 habitantes (IBGE, 2014).

A pesquisa foi realizada em todas as unidades de saúde existentes no município. Neste há cinco Unidades Básicas de Saúde (duas na cidade e três na zona rural) e uma Unidade Mista de Saúde. Decidiu-se incluir todas as unidades visto que nelas há realização de procedimentos como administração de vacinas, medicações, testes de glicemia capilar, curativos, retirada de pontos, exame colpocitológico, entre outros. Procedimentos mais complexos são realizados em unidades de referência localizadas em municípios maiores do estado do Pernambuco. A coleta dos dados ocorreu no período correspondente ao mês de abril de 2015.

Os participantes do estudo foram os enfermeiros e técnicos de enfermagem que prestam assistência de enfermagem à população nos serviços de saúde do município. Todos os profissionais foram abordados, explicando-se os objetivos da 
pesquisa e os procedimentos para coleta de dados, através do Termo de Consentimento Livre e Esclarecido. Os participantes tiveram suas identidades preservadas, sendo estes identificados pela letra $P$, representando Profissional, seguido de número $(1,2,3 \ldots)$ inseridos aleatoriamente.

Para obtenção de informações, foi aplicado um questionário, previamente elaborado pelos pesquisadores. O questionário foi entregue, disponibilizou-se de tempo para a retirada de dúvidas em relação às perguntas e foi agendado um horário ou dia para que os profissionais devolvessem o instrumento preenchido.

A análise de dados foi realizada através da análise de conteúdo, submetendose as respostas coletadas através do questionário a três fases: a fase de préanálise, na qual houve leitura das respostas contidas nos questionários; em seguida, a exploração do material, compreendendo a observação e detalhamento dos principais pontos respondidos; e, para que os dados fossem agregados, fez-se necessário a categorização dos mesmos, consistindo na terceira fase do processo (GOMES, 1999).

A pesquisa seguiu os termos da Resolução n $466 / 12$ do Conselho Nacional de Saúde (Brasil, 2012), tendo aprovação do Comitê de Ética em Pesquisa da Faculdade Leão Sampaio, com o parecer no 995.394.

\section{RESULTADOS E DISCUSSÃO}

Todos os profissionais da equipe de enfermagem foram abordados. No entanto, uma enfermeira não devolveu o questionário preenchido. Dessa forma, participaram do estudo seis enfermeiras e nove técnicas de enfermagem, todas mulheres e com idade variando entre 23 a 57 anos.

Quanto ao sexo, observa-se que, em pesquisas realizadas com a equipe de enfermagem, as mulheres costumam ser maioria, fato que se explica por ser a enfermagem uma profissão predominantemente feminina (Doi e Moura, 2011; Santos e Souza, 2012).

Inicialmente, perguntaram-se aos profissionais quais resíduos eram gerados nas unidades pesquisadas. As respostas deram origem a seguinte categoria:

\section{Tipos de resíduos gerados}

De acordo com a variedade de procedimentos e, levando-se em consideração as características das unidades pesquisadas, espera-se geração de resíduos que requeiram segregação e acondicionamento adequados. Obtiveram-se as seguintes respostas:

-P1 Perfurocortantes (agulhas, lâminas de bisturi); material contaminado (espéculo citológico, fita para uso de glicosímetro, material de curativo, entre 
outros); lixo comum; material reciclável (embalagens plásticas como, embalagem de soro, álcool e etc).

-P3 Infectantes, perfurocortantes.

-P5 Nessa unidade são produzidos os seguintes resíduos: resíduos químicos, resíduos comuns, resíduos biológicos e perfurocortantes.

-P6 Materiais resultantes de atividades humanas; materiais perfurocortantes; substâncias químicas e outros.

-P10 São agulhas, bisturi, seringas, gaze, luva, papel, frasco de soro seco, ampolas de injetáveis.

Nas respostas obtidas, notou-se que os procedimentos realizados pela equipe de enfermagem nos estabelecimentos de saúde pesquisados, geraram resíduos de diferentes classes.

Mesmo alguns dos profissionais não citando os resíduos gerados por agrupamento, percebeu-se que os resíduos identificados nos serviços de saúde pertencem a quatro diferentes grupos: resíduos biológicos, resíduos químicos, resíduos comuns e resíduos perfurocortantes, de acordo com a classificação da RDC no 306/04 da ANVISA e a Resolução n 358/05 do CONAMA (Brasil, 2004; Brasil, 2005).

Nas UBS pesquisadas, os resíduos gerados são os biológicos, comuns, químicos e perfurocortantes. Os locais de maior geração são a triagem, a sala de vacinas, a sala de procedimentos e o consultório de enfermagem. Nesses locais são realizadas atividades tais como: administração de vacinas, medicações, testes de glicemia capilar, curativos, retiradas de pontos de suturas e exame colpocitológico.

Na Unidade Mista de Saúde pesquisada, os pontos de maior geração de resíduos são as enfermarias e a sala de emergência, gerando resíduos biológicos, perfurocortantes e comuns. Em relação aos resíduos comuns, estes são gerados em todos os departamentos da Unidade.

Aos profissionais foi perguntado se realizavam o processo de segregação e acondicionamento nas suas unidades e de que forma realizavam esses procedimentos. As respostas levaram a duas categorias, descritas a seguir: saúde

Procedimentos adotados na segregação dos resíduos de serviços de

Os entrevistados responderam que realizavam a segregação, mas ao descrever a forma, esta nem sempre foi descrita de acordo com o que preconiza a legislação sobre gerenciamento de resíduos de serviços de saúde. É o que se pode perceber nas seguintes respostas:

-P2 Nesta unidade os resíduos são separados apenas em lixo comum e perfurocortantes. 
-P5 O processo utilizado nessa unidade se limita a divisão dos perfurocortantes dos demais. Sendo que tudo é levado pela vigilância como lixo hospitalar. Dividindo-o apenas em dois tipos: perfurocortante e lixo comum.

\section{Utilização de recipientes para o acondicionamento de resíduos de serviços de saúde}

Com relação ao processo de acondicionamento, as respostas dos profissionais mostraram que não há padronização dos recipientes, estes não são identificados da maneira preconizada pela legislação e alguns recipientes são utilizados a partir do improviso de caixas ou outro material. Essa categoria está representada a seguir:

-P15 Não são divididos em lixeiras diferentes na unidade, e não se usam sacolas apropriadas. Os pérfuros são armazenados em caixas improvisadas. A única divisão são dos comuns, e contaminados dos perfuros.

-P14 São acondicionados em recipientes comuns sem identificação de risco.

-P7 Os perfurocortantes são armazenados em caixa de papelão descartável. Quanto aos outros tipos de resíduos são armazenados em balde com tampa e os demais são armazenados em sacos plásticos.

-P10 No caso de perfurocortante guardamos em caixas de papelão, feitos por improviso.

Há uma distinta ausência de procedimentos padronizados no cotidiano das unidades básicas de saúde. A produção ambulatorial de procedimentos assistenciais se distancia do processo de adequação dos resíduos gerados e sua destinação socioambiental sustentável e segura.

De acordo com as respostas dos participantes, observou-se que o lixo comum e o lixo infectado são desprezados erroneamente no mesmo recipiente misturandose desta forma os resíduos biológicos, químicos e comuns. A única separação existente é a dos perfurocortantes, no entanto, em recipientes improvisados, dado coincidente com pesquisa realizada sobre o manejo de resíduos provenientes de cuidados domiciliares (ALVES et al., 2012).

Em uma pesquisa realizada com enfermeiras da Estratégia de Saúde da Família de três municípios do interior da Bahia, ao se perguntar se nas unidades de saúde havia o correto acondicionamento de resíduos, estas responderam que o procedimento não é feito de maneira adequada ou que desconhecem como ocorre o acondicionamento em suas unidades (SANTOS; SOUZA, 2012).

No Hospital das Clínicas de Porto Alegre, em estudo que teve como participantes profissionais da equipe de enfermagem, observou-se que a segregação e, consequentemente, o acondicionamento, também não é realizado de 
maneira adequada, sendo citado materiais como seringas, mantas e fraldas, sendo descartados em recipientes inapropriados para tais materiais (DOI; MOURA, 2011).

Em pesquisa realizada com equipes de enfermagem da atenção básica e farmacêuticos da assistência farmacêutica de um município baiano, também houve divergência entre as orientações e ações desses profissionais, quando se trata do descarte de medicamentos, muitos deles sendo descartados no lixo comum e, também, na rede de esgoto (ALENCAR et al., 2014).

Quando se ampliam os participantes da pesquisa para médicos e pessoal da limpeza, um terço dos profissionais pesquisados tem conhecimento deficiente sobre o manejo dos resíduos. Já quanto à prática, enfermeiros tem melhor desempenho e quase metade dos médicos tem prática deficiente na segregação de resíduos (SARKER et al., 2014).

Evidencia-se em todos os contextos e profissionais de saúde, que estes precisam ser envolvidos nas ações de manejo de resíduos. A equipe de enfermagem passa um tempo maior ao lado do paciente e realiza uma ampla variedade de procedimentos. No entanto, outros profissionais também manipulam resíduos em suas atividades e precisam ser envolvidos nas atividades de treinamento e educação para estarem informados sobre a legislação e sensibilizados com a causa ambiental. Esses treinamentos devem ser periódicos e fazer parte de um programa de educação continuada.

A segregação consiste na separação dos resíduos na sua fonte de geração, no momento em que se detecta a necessidade de descartá-los, levando em consideração a composição, as características físicas e os riscos provenientes de suas características e transmissibilidade de doenças (BRASIL, 2004; BRASIL, 2005).

A segregação dos resíduos na sua fonte de geração tem como benefícios a redução do volume destes, a diminuição dos riscos de acidentes e, também, o tratamento adequado quando se trata de resíduos infectados. Quando se mistura o resíduo comum com o contaminado, como relatado pelos profissionais entrevistados nessa pesquisa, estes passam a ser considerados como contaminados (PEREIRA et al., 2013; BRASIL, 2006; PAIZ et al., 2014).

Concomitantemente, aumentam os gastos dos serviços de saúde com o tratamento final, os riscos de acidentes pelos profissionais que os manipulam ou fazem o seu transporte, e os danos ao ambiente, quando estes não têm o tratamento e a disposição final adequada.

Os profissionais relataram que não há padronização e identificação dos recipientes utilizados para acondicionamento dos resíduos. Pesquisa realizada em João Pessoa, no estado da Paraíba, encontrou dado semelhante em 26,34\% das unidades de saúde visitadas (RAMOS et al., 2011).

Os resíduos devem ser acondicionados em recipientes padronizados para cada tipo de material, e identificados por símbolos com linguagem universal, que permita a identificação do resíduo a partir do que se observa nos rótulos e 
recipientes (DOI; MOURA, 2011; BRASIL, 2004). Essa medida visa a informar os riscos que os resíduos podem ter e a forma de paramentação adequada para quem vai transportar esse material seja no ambiente interno ou externo ao serviço gerador do resíduo.

A RDC no 306/04 da ANVISA prevê que os perfurocortantes devem ser descartados em recipientes rígidos, resistentes à punctura, ruptura e vazamento, observando-se 0 limite de preenchimento indicado, sendo proibido 0 reaproveitamento desses recipientes (BRASIL, 2004).

Outro fato que deve ser destacado a partir das respostas é o fato de que não existe separação específica para os resíduos comuns, além de que estes são desprezados junto com os resíduos contaminados. O erro mais comum encontrado é o acondicionamento conjunto de resíduos comuns e infectados (ADUAN et al., 2014), dado também encontrado em pesquisa realizada em Mafra, Santa Catarina (POVALUK, 2012).

A necessidade de se reduzir o volume de resíduos, parte do princípio de que estes, ao serem classificados como comuns, podem ser reutilizados ou reciclados. A redução do volume é um ponto importante para diminuir o impacto ambiental (DOI; MOURA, 2011; BESEN et al., 2014).

No entanto, é insegura a possibilidade de reutilizar ou reciclar os resíduos das unidades pesquisadas, já que todos os resíduos não perfurocortantes estão sendo desprezados nos mesmos recipientes com probabilidade e risco para acidentes aumentados.

É necessário sensibilizar os profissionais para a redução do volume de resíduos, visto que, para o tratamento final dos resíduos infectantes, o que geralmente é feito por incineração, os serviços de saúde pagam um valor calculado a partir do peso dos resíduos. Se há mistura de resíduo comum com contaminado, então os serviços de saúde estão pagando um valor muito maior do que se houvesse descarte apenas dos infectantes.

A separação adequada dos resíduos comuns permite a reciclagem e 0 reaproveitamento destes, poupando o ambiente de um grande volume de produtos tóxicos e de difícil degradação.

O adequado manejo dos resíduos de serviços de saúde parte de uma preocupação inicial com a preservação dos recursos ambientais. Isso se repete em vários países de forma que, de maneira conjunta, discutiram-se e traçaram-se metas mundiais, visando à proteção ambiental. Esses países passaram a regulamentar o gerenciamento de resíduos através de legislações próprias.

Em pesquisa realizada em Teerã, capital do Irã, observou-se que há manejo adequado do lixo hospitalar nas etapas de armazenamento temporário e transporte interno e externo, mas inadequado na segregação e tratamento final. Outro dado observado é que, naqueles hospitais onde havia especialista em saúde ambiental, os processos aconteciam de forma mais adequada. Esta pesquisa indicou também, 
programas de treinamentos para os profissionais, como sugestão para melhoria do manejo dos resíduos (MALEKAHMADI et al., 2014).

Pesquisa no Reino Unido mostrou que um ano após a realização de uma auditoria interna, o gerenciamento de resíduos hospitalares continuava deficiente, com segregação inadequada, carrinhos de transporte em áreas acessíveis ao público e áreas de armazenamento em mau estado de conservação, apesar da legislação existente (BLENKHARN, 2007).

No que tange a forma pela qual os profissionais das unidades acondicionam seus resíduos, é notória a incoerência com as normas estabelecidas, evidenciandose um acondicionamento precário de acordo com as normas e leis que tratam do manejo de RSS.

\section{CONSIDERAÇÕES FINAIS}

Os procedimentos adotados pelos profissionais na segregação e acondicionamento de RSS foram identificados e considerou-se que estes não são feitos de maneira adequada. Há mistura de resíduo comum com infectado. Os resíduos perfurocortantes são separados, no entanto, os recipientes são improvisados e não tem identificação.

O presente estudo não identificou as causas das inadequações do gerenciamento dos resíduos. Visto que estas podem ser múltiplas, desde o desconhecimento dos profissionais quanto ao procedimento correto até a falta de equipamentos adequados, sugere-se que outros estudos sejam feitos para investigar essas causas.

De qualquer forma, percebe-se que há necessidade de capacitar os profissionais sobre essa temática, trabalhando-se a legislação, em especial a

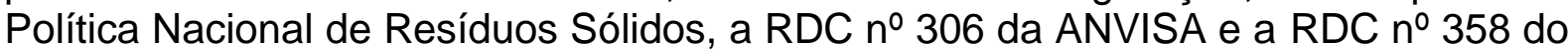
CONAMA.

\section{REFERÊNCIAS}

ADUAN, S. A. et al. Avaliação dos resíduos de serviços de saúde do Grupo A em hospitais de Vitória (ES), Brasil. Eng. Sanit. Ambient., Rio de Janeiro, v. 19, n. 2, p. 133-141, jun. 2014. Disponível em <http://www.scielo.br/scielo.php?script= sci_arttext\&pid=S1413-41522014000200133\&lng=pt\&nrm=iso>. Acesso em: 18 jul. 2015. http://dx.doi.org/10.1590/S1413-41522014000200004. 
ALENCAR, T. de O. S. et al. Descarte de medicamentos: uma análise da prática no Programa Saúde da Família. Ciênc. saúde coletiva, Rio de Janeiro, v. 19, n. 7, p. 2157-2166, jul. 2014. Disponível em: <http://www.scielo.br/scielo.php? script=sci_arttext\&pid=S1413-81232014000702157\&lng=pt\&nrm=iso. Acesso em: 18 jul. 2015. http://dx.doi.org/10.1590/1413-81232014197.09142013.

ALVES, S. B. et al. Manejo de resíduos gerados na assistência domiciliar pela Estratégia de Saúde da Família. Rev. bras. enferm., Brasília, v. 65, n. 1, p. 128-134, fev. 2012. Disponível em <http://www.scielo.br/scielo.php?script= sci_arttext\&pid=S0034-71672012000100019\&lng=pt\&nrm=iso >. Acesso em: 18 jul. 2015. http://dx.doi.org/10.1590/S0034-71672012000100019.

BAPTISTINI, R. A.; FIGUEIREDO, T. A. M. de. Agente comunitário de saúde: desafios do trabalho na zona rural. Ambient. soc., São Paulo, v. 17, n. 2, p. 53-70, jun. 2014. Disponível em: <http://www.scielo.br/scielo.php?script=sci_arttext\&pid= S1414-753X2014000200005\&lng=pt\&nrm=iso >. Acesso em: 18 jul. 2015. http://dx.doi.org/10.1590/S1414-753X2014000200005.

BASU, M.; DAS, P.; PAL, R. Assessment of future physicians on biomedical waste management in a tertiary care hospital of West Bengal. J Nat Sci Biol Med, v. 3, n. 1, p. 38-42, Jan 2012. ISSN 2229-7707. Disponível em: <http://www.ncbi.nlm.nih.gov/pubmed/22690049>.

BESEN, G. R. et al. Coleta seletiva na Região Metropolitana de São Paulo: impactos da Política Nacional de Resíduos Sólidos. Ambient. soc., São Paulo, v. 17, n. 3, p. 259-278, set. 2014. Disponível em: <http://www.scielo.br/scielo.php?script= sci_arttext\&pid=S1414-753X2014000300015\&lng=pt\&nrm=iso>. acessos em 18 jul. 2015. http://dx.doi.org/10.1590/S1414-753X2014000300015.

BLENKHARN, J. I. Standards of clinical waste management in hospitals--a second look. Public Health, v. 121, n. 7, p. 540-5, Jul 2007. ISSN 0033-3506. Disponível em: < http://www.ncbi.nlm.nih.gov/pubmed/17296210 >.

BRASIL. Ministério da Saúde. RDC n 306, de 07 de dezembro de 2004. Dispõe sobre o regulamento técnico para o gerenciamento de resíduos de serviços de saúde. Diário Oficial da União, 10 de dezembro de 2004.

BRASIL. Ministério do Meio Ambiente e da Amazônia Legal. Resolução CONAMA no 358, de 29 de abril de 2005. Diário Oficial da União, 4 de maio de 2005. Seção 1 , p. 63-65.

BRASIL. Ministério da Saúde. Agência Nacional de Vigilância Sanitária. Manual de gerenciamento de resíduos de serviços de saúde. Ministério da Saúde, Agência Nacional de Vigilância Sanitária. Brasília: Ministério da Saúde, 2006.

BRASIL. Presidência da República. Lei no 12.305, de 02 de agosto de 2010. Institui a Política Nacional de Resíduos Sólidos, altera a Lei n- 9.605, de 12 de fevereiro de 1998; e dá outras providências. Diário Oficial da União, 3 ago. 2010. 
BRASIL. Conselho Nacional de Saúde. Resolução no 466, de 12 de dezembro de 2012. Dispõe sobre diretrizes e normas regulamentadoras de pesquisas envolvendo seres humanos. Brasília: Ministério da Saúde, 2012.

DOI, K. M.; MOURA, G. M. S. S. de. Resíduos sólidos de serviços de saúde: uma fotografia do comprometimento da equipe de enfermagem. Rev. Gaúcha Enferm. (Online), Porto Alegre, v. 32, n. 2, p. 338-344, jun. 2011. Disponível em: <http://www.scielo.br/scielo.php?script=sci_arttext\&pid=S1983$14472011000200018 \&$ Ing=pt\&nrm=iso >. Acesso em: 18 jul. 2015. http://dx.doi.org/10.1590/S1983-14472011000200018.

GOMES, R. A análise de dados em pesquisa qualitativa. In MINAYO, Maria Cecília de Sousa. Pesquisa Social: Teoria, método e criatividade. 14 ed. Petrópolis: Vozes, 1999.

GONCALVES, E. M. do N. et al . Modelo de implantação de plano de gerenciamento de resíduos no laboratório clínico. J. Bras. Patol. Med. Lab., Rio de Janeiro , v. 47, n. 3, p. 249-255, jun. 2011. Disponível em: <http://www.scielo.br/scielo.php? script=sci_arttext\&pid=S1676-24442011000300008\&lng=pt\&nrm=iso >. Acesso em: 19 jul. 2015. http://dx.doi.org/10.1590/S1676-24442011000300008.

IBGE. Instituto Brasileiro de Geografia e Estatística. Banco de Dados. Disponível em:<http://www.cidades.ibge.gov.br/xtras/perfil.php?lang=\&codmun=260430>. Acesso em: 22 fev. 2014.

MALEKAHMADI, F. et al. Analysis of the healthcare waste management status in Tehran hospitals. J Environ Health Sci Eng, v. 12, n. 1, p. 116, 2014. ISSN 2052336X. Disponível em: <http://www.ncbi.nlm.nih.gov/pubmed/25426295>.

MARCHI, V. et al. Conhecimento dos pacientes do SUS sobre o gerenciamento de resíduos gerados em suas residências. Saúde Meio Ambient., v. 2, n. 2, p. 98-106, dez. 2013. Disponível em <http://www.periodicos.unc.br/index.php/ sma/article/view/521/398>. Acesso em: 08 dez. 2015.

NASCIMENTO, T. C. et al. Ocorrência de bactérias clinicamente relevantes nos resíduos de serviços de saúde em um aterro sanitário brasileiro e perfil de susceptibilidade a antimicrobianos. Rev. Soc. Bras. Med. Trop., Uberaba, v. 42, n. 4, p. 415-419, ago. 2009. Disponível em: <http://www.scielo.br/scielo.php?script= sci_arttext\&pid=S0037-86822009000400011\&lng=pt\&nrm=iso >. Acesso em: 18 jul. 2015. http://dx.doi.org/10.1590/S0037-86822009000400011.

PAIZ, J. C. et al. Aplicação de gráficos nightingaleanos para avaliação da heterogeneidade de Resíduos de Serviço de Saúde de um hospital. Rev. LatinoAm. Enfermagem, Ribeirão Preto, v. 22, n. 6, p. 942-949, dez. 2014. Disponível em: $<$ http://www.scielo.br/scielo.php?script=sci_arttext\&pid=S0104-

11692014000600942\&lng=pt\&nrm=iso>. Acesso em: 18 jul. 2015. Epub 16-Dez2014. http://dx.doi.org/10.1590/0104-1169.3309.2499. 
PEREIRA, M. S. et al. Gerenciamento de resíduos em unidades não hospitalares de urgência e emergência. Rev. Latino-Am. Enfermagem, Ribeirão Preto, v. 21, n. spe, p. 259-266, fev. 2013. Disponível em:

<http://www.scielo.br/scielo.php?script=sci_arttext\&pid=S0104-

$11692013000700032 \&$ Ing=pt\&nrm=iso >. Acesso em: 17 jul. 2015.

http://dx.doi.org/10.1590/S0104-11692013000700032.

POVALUK, M. Educação ambiental x sustentabilidade: proposta de um plano de gerenciamento de resíduos de serviços de saúde. Saúde Meio Ambient., v. 1, n. 1, jun. 2012. Disponível em <http://www.periodicos.unc.br/index.php/ sma/article/view/220/263 >. Acesso em: 08 dez. 2015.

RAMOS, Y. S. et al. Vulnerabilidade no manejo dos resíduos de serviços de saúde de João Pessoa (PB, Brasil). Ciênc. saúde coletiva, Rio de Janeiro, v. 16, n. 8, p. 3553-3560, ago. 2011 . Disponível em: <http://www.scielo.br/scielo.php?script=sci_ arttext\&pid=S1413-81232011000900023\&lng=pt\&nrm=iso >. Acesso em: 18 jul. 2015. http://dx.doi.org/10.1590/S1413-81232011000900023.

SALES, C. C. de L. et al. Gerenciamento dos resíduos sólidos dos serviços de saúde: aspectos do manejo interno no município de Marituba, Pará, Brasil. Ciênc. saúde coletiva, Rio de Janeiro, v. 14, n. 6, p. 2231-2238, dez. 2009. Disponível em: <http://www.scielo.br/scielo.php?script=sci_arttext\&pid=S1413-

$81232009000600032 \&$ Ing=pt\&nrm=iso >. Acesso em: 17 jul. 2015. http://dx.doi.org/10.1590/S1413-81232009000600032.

SARKER, M. A. et al. Evaluation of knowledge, practices, and possible barriers among healthcare providers regarding medical waste management in Dhaka, Bangladesh. Med Sci Monit, v. 20, p. 2590-7, 2014. ISSN 1643-3750. Disponível em: < http://www.ncbi.nlm.nih.gov/pubmed/25488747>.

SANTOS, M. A. dos; SOUZA, A. de O. Conhecimento de enfermeiros da Estratégia Saúde da Família sobre resíduos dos serviços de saúde. Rev. bras. enferm., Brasília , v. 65, n. 4, p. 645-652, ago. 2012. Disponível em: <http://www.scielo.br/scielo.php?script=sci_arttext\&pid=S0034$71672012000400014 \& \mathrm{lng}=$ pt\&nrm=iso >. Acesso em: 18 jul. 2015. http://dx.doi.org/10.1590/S0034-71672012000400014.

SCHOLZ, S.; NGOLI, B.; FLESSA, S. Rapid assessment of infrastructure of primary health care facilities - a relevant instrument for health care systems management.

BMC Health Serv Res, v. 15, p. 183, 2015. ISSN 1472-6963. Disponível em: <http://www.ncbi.nlm.nih.gov/pubmed/25928252>.

Artigo recebido em: 17/12/2015

Artigo aprovado em: 29/11/2016 